\title{
AN INTEGRAL ANALOGUE TO PARALLLOGRAM LAW
}

\author{
A. J. PENICO AND Č. V. STANOJEVIĆ
}

ABsTract. It is shown that there is an integral analogue to the classical characterization of inner product spaces. The sharp bounds for the integral of the norm are found.

1. Let $N$ be a normed complex linear space, $S$ its unit sphere, and let

$$
\Phi(x, y)=\frac{1}{2 \pi} \int_{0}^{2 \pi}\|(\cos t) x+(\sin t) y\|^{2} d t .
$$

In [1] it was announced that $N$ is an inner product space if and only if $\Phi(x, y)<1$, for all $x$ and $y$ in $S$. The purpose of this paper is to prove the above characterization of inner product spaces and give best possible bounds for (1).

2. The following theorem is an integral analogue to the Schoenberg version [2] of the classical M. M. Day result [3].

TheOREM 1. Let $N$ be a normed complex linear space and $S$ its unit sphere. Then $N$ is an inner product space if and only if

$$
\Phi(x, y)<1 \text { for all } x, y \in S .
$$

Proof. The "only if" part is immediate. For the "if" part it suffices to consider a 2-dimensional normed real linear space $N_{2}$. Let $S_{2}$ be the unit sphere of $N_{2}$. In [3] it was shown (a result attributed to Loewner) that $S_{2}$ can be encompassed by a unique minimal ellipse $E$ which meets $S_{2}$ in four distinct points, say, $x, y,-x,-y$, with $x$ and $y$ being linearly independent. The ellipse induces in $N_{2}$ a euclidean norm $\|\cdot\|_{E}$, with $E$ as a unit sphere. Hence $\|Z\|_{E}<\|Z\|$ for all $z \in N_{2}$. Let $x$ and $y$ be as chosen above. Then $\|x\|=\|y\|=\|x\|_{E}=\|y\|_{E}=1$, and

$$
1=\frac{1}{2 \pi} \int_{0}^{2 \pi}\|(\cos t) x+(\sin t) y\|_{E}^{2} d t<\Phi(x, y)<1,
$$

whence

$$
\int_{0}^{2 \pi}\|(\cos t) x+(\sin t) y\|_{E}^{2} d t=\int_{0}^{2 \pi}\|(\cos t) x+(\sin t) y\|^{2} d t
$$

Received by the editors April 5, 1979 and, in revised form, July 9, 1979.

AMS (MOS) subject classifications (1970). Primary $46 \mathrm{Cl0}$.

Key words and phrases. Inner product spaces. 
For fixed $x$ and $y$ and for all $t$, from the continuity and nonnegativity of

$$
\|(\cos t) x+(\sin t) y\|^{2}-\|(\cos t) x+(\sin t) y\|_{E}^{2},
$$

it follows that

$$
\|(\cos t) x+(\sin t) y\|=\|(\cos t) x+(\sin t) y\|_{E}
$$

Since every $z \in N_{2}$ is of the form

$$
z=(\rho \cos t) x+(\rho \sin t) y, \quad \rho>0,
$$

we have

$$
\|z\|=\|z\|_{E}
$$

for all $z \in N_{2}$.

This proves the theorem.

3. The sharp bounds of (1) are established in the next theorem.

THEOREM 2. Let $N$ be a normed complex linear space and $S$ its unit sphere. Then

$$
\frac{1}{2}+\frac{1}{\pi}<\Phi(x, y)<2\left(\frac{1}{2}+\frac{1}{\pi}\right)
$$

for all $x, y \in S$.

Proof. From

$$
\Phi(x, y)=\frac{1}{\pi} \int_{0}^{\pi}\|(\cos t) x+(\sin t) y\|^{2} d t
$$

it follows that

$$
\begin{aligned}
\pi \Phi(x, y) & <\int_{0}^{\pi}[\|(\cos t) x\|+\|(\sin t) y\|]^{2} d t \\
& <\frac{\pi}{2}\left[\|x\|^{2}+\|y\|^{2}\right]+\|x\|\|y\| \int_{0}^{\pi}|\sin t| d t \\
& =\frac{\pi}{2}\left[\|x\|^{2}+\|y\|^{2}\right]+2\|x\|\|y\| .
\end{aligned}
$$

Finally,

$$
\begin{aligned}
\Phi(x, y) & <\frac{1}{2}\left[\|x\|^{2}+\|y\|^{2}\right]+\frac{2}{\pi}\|x\|\|y\| \\
& =1+\frac{2}{\pi}=2\left(\frac{1}{2}+\frac{1}{\pi}\right),
\end{aligned}
$$

for all $x, y \in S$.

On the other hand, 


$$
\begin{aligned}
\pi \Phi(x, y)= & \int_{0}^{\pi}\|(\cos t) y+(\sin t) y\|^{2} d t \\
= & \int_{0}^{\pi / 2}\|(\cos t) x+(\sin t) y\|^{2} d t+\int_{\pi / 2}^{\pi}\|(\cos t) x+(\sin t) y\|^{2} d t \\
& =\int_{0}^{\pi / 2}\left[\|(\cos t) x+(\sin t) y\|^{2}+\|(-\sin t) x+(\cos t) y\|^{2}\right] d t \\
& =\frac{1}{2} \int_{-\pi / 4}^{\pi / 4}\left[\|(\cos t-\sin t) x+(\sin t+\cos t) y\|^{2}\right. \\
& \left.+\|(\cos t+\sin t) x-(\cos t-\sin t) y\|^{2}\right] d t
\end{aligned}
$$

Or,

$$
\pi \Phi(x, y) \geqslant \int_{0}^{\pi / 4}(\sin t+\cos t)^{2} d t\left[\|x\|^{2}+\|y\|^{2}\right]=\left(\frac{\pi}{4}+\frac{1}{2}\right)\left[\|x\|^{2}+\|y\|^{2}\right] .
$$

Finally,

$$
\Phi(x, y)>\frac{1}{2}+\frac{1}{\pi}
$$

for all $x, y \in S$.

It remains to show that the above bounds are best possible. Indeed, consider the 2-dimensional normed linear space

$$
l_{2}^{2}=\left\{\left(x_{1}, x_{2}\right) \mid x_{1}, x_{2} \in \mathbf{R}\right\}
$$

with

$$
\left\|\left(x_{1}, x_{2}\right)\right\|=\left|x_{1}\right|+\left|x_{2}\right|
$$

Then

$$
\Phi((1,0),(0,1))=\frac{1}{\pi} \int_{0}^{\pi}(|\cos t|+|\sin t|)^{2} d t=1+\frac{2}{\pi}
$$


and

$$
\begin{aligned}
\Phi\left(\left(\frac{1}{2}, \frac{1}{2}\right),\left(\frac{1}{2}, \frac{1}{2}\right)\right) & =\frac{1}{\pi} \int_{0}^{\pi} \frac{1}{4}[|\cos t+\sin t|+|\cos t-\sin t|]^{2} d t \\
& =\frac{1}{2}+\frac{1}{\pi} .
\end{aligned}
$$

It would be of interest to study more general subharmonic properties of norms, other than (2), and relate them to the problem of characterization of inner-product spaces.

\section{REFERENCES}

1. A. J. Penico and C. V. Stanojevic, Some characterizations of inner-product spaces, Notices Amer. Math. Soc. 24 (1977), A-123.

2. I. J. Schoenberg, A remark on $M$. M. Day's characterization of inner-product spaces and a conjecture of L. M. Blumenthal, Proc. Amer. Math. Soc. 3 (1952), 961-964.

3. M. M. Day, Some characterizations of inner-product spaces, Trans. Amer. Math. Soc. 62 (1947), 320-337.

Departmient of Mathematics, University of Missouri-Rolla, Rolla, Missouri 65401 